InnOvaciOnes de NegOciOs 17(34): 234-253

(c) 2019 UANL, Impreso en México (ISSN: 2007-1191)

Recepción: 8 julio de 2020 Aceptación: 16 julio de 2020

\title{
Guías de diseño para generar flujo de trabajo en proceso de compras (Design lines to generate workflow in purchasing process)
}

\author{
Cristel Fragoso Pacheco, Gilberto Borrego, Ramón Palacio Cinco y \\ Mario A. Núñez \\ Instituto Tecnológico de Sonora \\ cristel.fragos0208111@potros.itson.edu.mx
}

\begin{abstract}
Purchase management is the process which responds to the changing demands of the environment, resulting in increased profits for companies. Specifically, purchases in health institutions are becoming more relevant, since these institutions have the challenge of serving with quality, providing timely responses, ensuring security in their purchases, acting efficiently and being in constant innovation. The context in which purchases for health institutions involves growing demand, rapid technological evolution and limited economic and financial resources. Workflow system is a highly supportive tool for the management of any process, it consists of focusing on the procedure of each work activity assigning resources and tasks, making the work more efficient through improving procedures. The main objective of this document is proposal of through the a case study carried out with PADM (Process Analysis Design Methodology) and an analysis of tools found in the market, a design guide to implement a workflow system in the private clinics purchasing process that reduces time and costs.
\end{abstract}

Key words: BPM, Design guide, Purchase management, Workflow.

JEL: D23, 03,14, O30, O31

Resumen. La gestión de compras puede responder a las demandas cambiantes del entorno y resultar en un incremento en los beneficios para las empresas. Específicamente las compras en instituciones de salud cada vez adquieren mayor relevancia, ya que tienen el reto de atender con calidad, brindar respuestas oportunas, contar con seguridad en sus compras, actuar con eficiencia y estar en constante innovación, teniendo en cuenta el contexto de demanda creciente y exigente, una rápida evolución tecnológica, con recursos económicos y financieros generalmente limitados. El sistema flujo de trabajo es una herramienta de gran apoyo para la gestión de cualquier proceso, consiste en enfocarse en el procedimiento de cada actividad de trabajo asignando recursos y tareas, logrando que el trabajo sea más eficiente a través de la mejora de los procedimientos. Es por ello que el presente documento es un estudio de caso llevado a cabo mediante una metodología no

Guías de diseño para generar flujo de trabajo 
experimental, la utilizada es PADM (Process Analysis Design Methodology) y un análisis de herramientas que se encuentran en el mercado el cual propone una guía de diseño para implementar un flujo de trabajo en el proceso de compras de clínicas privadas que disminuya tiempos y costos.

Palabras clave: BPM, Flujo de trabajo, Gestión de compras, Guía de diseño.

\section{Introducción}

El comportamiento de los mercados presenta cambios constantes, lo cual obliga a las empresas a crear estructuras más flexibles para adaptarse a esos cambios, para ello deben prepararse y poder suministrar lo que los clientes demanden (Montoya, 2002). La sistematización de los procesos de las empresas, es una labor de gran impacto, de la que se puede ocupar eficazmente con la tecnología informática, desarrollando sistemas como el de flujo de trabajo conocido como workflow. El concepto de flujo de trabajo se originó pensando en la automatización de fabricación y de labores de oficinas, la idea es enfocarse en el procedimiento de cada actividad de trabajo asignando recursos y tareas, logrando que el trabajo sea más eficiente a través de la mejora de los procedimientos (Jiang et al., 2015).

Por su parte Salamanca y Gravito exponen un aplicativo informático que se basa en metodología Business Process Managment (BPM)(Jiménez et al., 2007), el cual J. Freund, B. Rücker y Hitpass lo definen como una "Disciplina de gestión por procesos de negocio y de mejora continua apoyada fuertemente por tecnología de información" (Freund et al., 2017) , Salas y Campoverde agregan que flexibiliza el modelado y se facilita la comprensión y automatización del proceso (Salas \& Campoverde, 2019); y el workflow, dónde muestra los múltiples beneficios que se obtuvieron al implementar ambos en el aplicativo informático, tales como incremento en la productividad de su capital humano, eliminando actividades repetitivas en la gestión de documentos, incremento en la efectividad y reducción en el tiempo de sus procesos administrativos, resultando en una satisfacción de las personas involucradas (Jiménez et al., 2007).

A parte de la metodología y el aplicativo antes mencionados, también existen plataformas y aplicaciones que brindan soporte al proceso de compras, así como software en la nube para digitalizar y centralizar la información del departamento de compras. Las plataformas y aplicaciones ayudan a simplificar y automatizar la gestión diaria del proceso, a disponer de 
herramientas de análisis y negociación con proveedores, para obtener mejores resultados y optimización del tiempo de los procesos. Algunas de esas plataformas son BidDown (https://biddown.com), Yaydoo (https://www.yaydoo.com/es), Monday (https://www.monday.com, y Wrike (https://www.wrike.com/es).

Una buena gestión de las compras puede responder a demandas cambiantes y resultar en un incremento de los beneficios en la empresa (Andino, 2006). El sector salud no es ajeno a esta realidad de demandas cambiantes, por lo que las compras hospitalarias cada vez adquieren una mayor relevancia en los planes y estrategias de dichas instituciones, ya que tienen el reto de atender con calidad, brindar respuestas oportunas, contar con seguridad en sus compras, actuar con eficiencia y estar en constante innovación, teniendo en cuenta el contexto de demanda creciente y exigente, una rápida evolución tecnológica, y con recursos económicos y financieros generalmente limitados (Camacho et al., 2012). Es por ello que se necesita de aplicaciones cada vez más complejas y destinadas a usuarios con más exigencia y con perfiles más variados, la personalización de las aplicaciones y su adaptación en función de la plataforma 0 del contexto en las que se ejecutan, se encuentran entre los requisitos principales a cumplir (InglésRomero \& Vicente-Chicote, 2012).

Para la creación de dichas aplicaciones, los desarrolladores pueden utilizar un documento llamado guías de diseño que les sirve de soporte en la construcción de los sitios web (Beltrán \& García, 2018). El objetivo de este documento es plasmar una guía de diseño con los principales componentes de un workflow para el proceso de compras hospitales privados.

El resto del artículo se estructura de la siguiente forma. En la sección 2 se presenta una breve revisión de literatura sobre el sistema workflow. La sección 3 describe el método utilizado para llegar a la guía de diseño. La sección 4 presenta los resultados obtenidos con respecto al método y con base en ellos, la guía de diseño resultante. Por último en la sección 5 se proporcionan algunos comentarios finales y futuras investigaciones.

\section{Marco teórico}

Actualmente nos encontramos en un entorno volátil, incierto, complejo y ambiguo, el cual da pie a un entorno competitivo donde debe haber una

Fragoso, C., Borrego, G., Palacio R. \& Núñez, M. 
adaptación tecnológica para poder prosperar en estos cambios del ambiente en tiempo y forma (Haposan et al., 2020). Dhir y Mital (2013) proponen que las organizaciones deben tomar continuamente ventaja de las oportunidades de mercado en evolución, y reaccionar rápidamente a cualquier cambio o evoluciones en el mercado (Dhir, 2018). Es por ello que se necesita de sistemas y tecnologías complementarias para los procesos, que aporten un carácter dinámico, y que proporcione un flujo constante durante el desarrollo de las actividades, y se trata de los workflow, herramientas que automatizan los procesos de la organización (Rodriguez \& González, 2002).

Un estudio realizado sobre la gestión de compras de una cooperativa agraria (Gómez, 2013), aborda el tema de la implementación de workflow para mejorar la gestión de compras de suministros, automatizando los procesos de trabajo y generando control en las diferentes etapas donde los documentos y la información se mueven entre los involucrados del proceso. Los beneficios que muestran son reducción de costos, pues al evitar los tiempos muertos con la automatización de las tareas relevantes, se aumenta el rendimiento de los trabajadores, además, las tareas costosas como la búsqueda, comparativa, análisis y envío de documentos pasa ahora a manos del sistema workflow.

Por su parte Orellana (en su trabajo de tesis menciona que Videnza Consultores (2018), observaron costos ocultos causados por la falta de planeación en la programación de las compras, que, a su vez, ocasionaron retrasos en la preparación, afectando la etapa de ejecución ya que se generan retrasos en las emisiones de órdenes de compras y en los pagos (Orellana, 2018).

De lo anterior expuesto, resalta la importancia de adaptarse al comportamiento del entorno con un sistema workflow que automatiza los procesos, aumentando los beneficios reduciendo costos, aumentado el rendimiento de trabajadores, eliminando retrasos, sólo por mencionar algunos en términos generales. Uno de los trabajos más relacionados colecta definiciones del significado de workflow por parte de varios autores y mencionan los múltiples beneficios del sistema, el antes mencionado trabajo de Gómez (2013) ese enfoca en mejorar la gestión del proceso de compras de una cooperativa agraria de España por medio de workflow. Sin embargo, no se encontró ninguna guía de diseño en la cual los desarrolladores de software que den soporte en los hospitales puedan apoyarse para 
implementar el sistema workflow como herramienta para facilitar la automatización del proceso de compras adoptando las medidas indicadas a partir de un previo análisis. El contar con guías de diseño sería un recurso muy útil para los diseñadores ya que en ella encontrarían detalladamente puntos relevantes a incluir en el sistema.

\section{Método}

En esta sección se presenta la metodología utilizada en este estudio de caso para obtener las guías de diseño. El alcance dentro de este artículo es llegar a la propuesta de las guías de diseño, para lograrlo se hizo uso de una metodología no experimental, en la que se observa el fenómeno, en este caso el proceso, tal como se da en su contexto natural para ser analizado (Hernández et al., 2014). La metodología se divide en dos etapas, en la primera etapa se aplica la metodología para el análisis y diseño de procesos (PADM- Process Analysis Design Methodology) con la que se analizará cada una de las actividades del proceso de compras, tiempo total del proceso y el costo con respecto a horas hombre en una jornada laboral, y en la segunda etapa se realiza un análisis de plataformas existentes en el mercado.

La metodología PADM cuenta con un marco extensible, flexible, y por lo tanto adaptable, consta de 4 fases (Martinez \& Cegarra, 2014):

1. Captura

2. Modelado

3. Análisis y evaluación

4. Rediseño

\section{Sujeto}

El sujeto de estudio de este proyecto, se enfoca al área de compras de una clínica hospital privada, específicamente en sus procesos de compras de cuadro básico y compras especiales, dónde en la gestión de dichas compras se maneja diariamente diferentes cantidades de información física y digital, sus procesos y procedimientos no están estandarizados ni documentados, por lo que se considera necesaria la implementación de un sistema Workflow.

Fragoso, C., Borrego, G., Palacio R. \& Núñez, M. 
La información fue proporcionada por el gerente de compras, el director administrativo, el almacenista y por la persona responsable de auditoría interna.

\section{Materiales}

Los materiales y herramientas necesarias para recolectar la información del proceso en estudio fueron los siguientes:

- Entrevistas semi-estructuradas, donde se pregunta a detalle por las actividades que comprenden los procesos de compras y compras especiales, las personas que intervienen, los roles, y las principales problemáticas que se presentan.

- Sistema modelador Bizagi (https://www.bizagi.com). Bizagi es un modelador de procesos que permite representar de forma esquemática todas las actividades y decisiones que se toman en el negocio. Con una interfaz que recuerda a Microsoft Office, Bizagi Process Modeler cumple con el estándar BPMN (Business Process Management Notation). Es gratuito, intuitivo y potente software de modelamiento de procesos.

\section{Procedimiento}

Una vez realizadas las reuniones iniciales con la empresa para expresar los intereses de cada una de las partes y establecer el acuerdo de colaboración, el procedimiento seguido en la primera etapa fue de acuerdo a las fases de la metodología PADM:

1) Captura: En esta etapa se obtiene información del proceso, del cómo se lleva a cabo, con herramientas como entrevistas semiestructuradas, observación, y obtención de documentos relevantes al proceso. De esta etapa se obtiene la descripción a detalle del proceso, en el que se identifican las responsabilidades de los involucrados y los tiempos de las actividades.

2) Modelado: Para modelar se usan técnicas diagramáticas, con el fin de analizar lo más relevante del proceso, como las actividades, su coordinación, el personal involucrado, los roles, los tiempos, interacciones, sistemas utilizados, etc. En esta etapa, se presenta el modelado del proceso a las personas involucradas para validar la 
información y corregir si es necesario. Las técnicas diagramáticas tienen un soporte por medio de herramientas de software para poder analizar los modelos, las técnicas utilizadas fueron Gráfica rica, Diagrama Rol-Actividad (RAD), IDEF0 y BPMN (Business Process Management Notation), éste último es el usado como base de estudio para correr una simulación en el software de Bizagi.

3) Análisis y evaluación: En esta etapa se verifica que el modelo describa correctamente el proceso. Es muy importarte realizar esta parte de la fase ya que sin un correcto análisis se podría tener información faltante, o incorrecta, lo que nos llevaría a tomar decisiones erróneas para el rediseño. Al realizar el análisis y la evaluación del proceso se podrán detectar actividades como duplicidad de información 0 tareas, 0 actividades irrelevantes. También es importante preguntar a los involucrados qué problemas detectan ellos, aquí se revisa el recurso humano, los tiempos, capacidad, estructura tecnológica, entre otros. El resultado final debe ser la comprensión de la necesidad de mejoría y el alcance de la mejoría, que signifique resultados satisfactorios para la empresa.

4) Rediseño y propuesta de mejora: Aquí los cambios o rediseños de actividades del proceso se hacen con el fin de proponer mejoras al mismo, en esta fase se proyectan primero mediante un modelado, las mejoras del proceso, principalmente eliminando actividades que no agregan valor, y se crea una simulación de la propuesta en bizagi para validarlo con el personal involucrado en el proceso.

Para la segunda etapa se seleccionaron aplicaciones que utilizan workflow, tomando como base las principales necesidades detectadas en la fase 4 de la primera etapa de la metodología, partiendo del análisis de las aplicaciones se realizó un aglomerado de las principales funciones que debe de tener el sistema workflow de un proceso de compras en hospitales privados para ser plasmados posteriormente en una guía de diseño.

Fragoso, C., Borrego, G., Palacio R. \& Núñez, M. 


\section{Resultados}

Análisis, evaluación y rediseño

Con la aplicación de la metodología PADM en este caso de estudio se obtuvieron los siguientes resultados.

El proceso inicia cuando se detecta la necesidad de que un bien 0 servicio debe ser adquirido y finaliza cuando se cumplen las obligaciones establecidas con los proveedores. Los procesos de compras se dividen en dos: compras de medicamento de cuadro básico, son fármacos que se pueden prescribir en la clínica con autorización del Consejo de Salubridad General (Instituto Mexicano del Seguro Social, s/f), y en compras especiales, las cuales son cualquier necesidad de compra fuera del medicamento de cuadro básico, pudiera ser equipo médico, mobiliario, instrumentos, etc.

El área de compras hace uso de un módulo entro del sistema ERP (Enterprise Resurce Planing), el cual se define como un "sistema de planificación de los recursos y de gestión de la información que de una forma estructurada puede satisfacer la demanda de las necesidades de la gestión empresarial" (Muñiz \& Prat, 2003), con ellos las empresas reducen costos y aumentan su eficiencia, estandarizando, racionalizando y agilizando los procesos de negocio (Conceição \& Gonzalez, 2010). El sistema ERP de la clínica llamado Sistema Hospital Integral (SHI), fue desarrollado por un empleado de la empresa, actualmente no cuenta con un módulo para compras, y no es compatible con dispositivos móviles. El área de compras hace uso de ventanas relacionadas con el módulo de inventario, también hace uso de una plataforma externa llamada Bionexo (https://beta.bionexo.com), la cual es un Marketplace de negocios para el área de salud, posee un conjunto de soluciones digitales que permiten a las instituciones de salud realizar la gestión online de todo su proceso de compras y abastecimiento, la clínica utiliza esta plataforma únicamente para solicitar cotizaciones y realizar algunas compras.

Las actividades identificadas en los procesos de compas y compras especiales se muestran en la Figura 1. También se obtuvieron las áreas 0 departamentos que en algún punto tienen participación con el proceso, las cuales se muestran en la Tabla 1. 
Tabla 1. Descripción de áreas que intervienen en el proceso de compras

\begin{tabular}{|c|c|}
\hline Área & Descripción \\
\hline $\begin{array}{l}\text { Cualquier área emisora de solicitud de } \\
\text { compra }\end{array}$ & $\begin{array}{l}\text { Enfermería, Administración, Dirección, } \\
\text { Laboratorio, Intendencia, Mantenimiento, } \\
\text { Farmacia }\end{array}$ \\
\hline Compras & $\begin{array}{l}\text { Gestionar la compra y adquisición de todos } \\
\text { los suministros con los que opera el hospital } \\
\text { (material médico quirúrgico, equipo médico, } \\
\text { materiales de papelería e intendencia, así } \\
\text { como los medicamentos que son de vital } \\
\text { importancia para nuestros pacientes). }\end{array}$ \\
\hline Almacén & $\begin{array}{l}\text { Recibe y revisa los materiales, repuestos, } \\
\text { equipo médico y otros suministros que } \\
\text { ingresan al almacén, acomodando los } \\
\text { materiales por fechas de caducidad evitando } \\
\text { tener suministros que estén próximos a } \\
\text { caducar. Surtiendo los materiales solicitados } \\
\text { por farmacia para el cumplimiento de las } \\
\text { necesidades de los pacientes. }\end{array}$ \\
\hline Dirección administrativa & $\begin{array}{l}\text { Planea, organiza y controla las actividades } \\
\text { de la empresa, con el objetivo de apoyar a } \\
\text { generar mayor rentabilidad, realizar toma de } \\
\text { decisiones en forma oportuna y confiable en } \\
\text { beneficio de la operación de la empresa. } \\
\text { Crea lineamientos de control, análisis } \\
\text { financiero, supervisar cumplimiento de } \\
\text { políticas, y sinergia con las demás áreas } \\
\text { operativas para lograr los objetivos. }\end{array}$ \\
\hline
\end{tabular}

Fuente: Elaboración propia.

Con la información obtenida de la fase de captura se modelaron los procesos de compras y compras especiales con diferentes técnicas, para fines de análisis se utilizaron los diagramas BPMN, los cuales se muestran en la Fig. 1 y Fig. 2.

Al realizar la simulación del proceso real de compras de cuadro básico, mostrado en la Figura 1, se encontró que el proceso tiene una duración de 3 días, 11 horas, 40 minutos, y un costo de horas hombre de $\$ 2,833.33$, al analizar esta parte del proceso se encontró con tiempo invertido en el tráfico de documentos en físico, inconsistencias en inventario, actividades manuales

Fragoso, C., Borrego, G., Palacio R. \& Núñez, M. 
que pudieran ser automatizadas, y tiempo de espera para la recepción de presupuestos.

Figura 1. Diagrama de proceso de compras

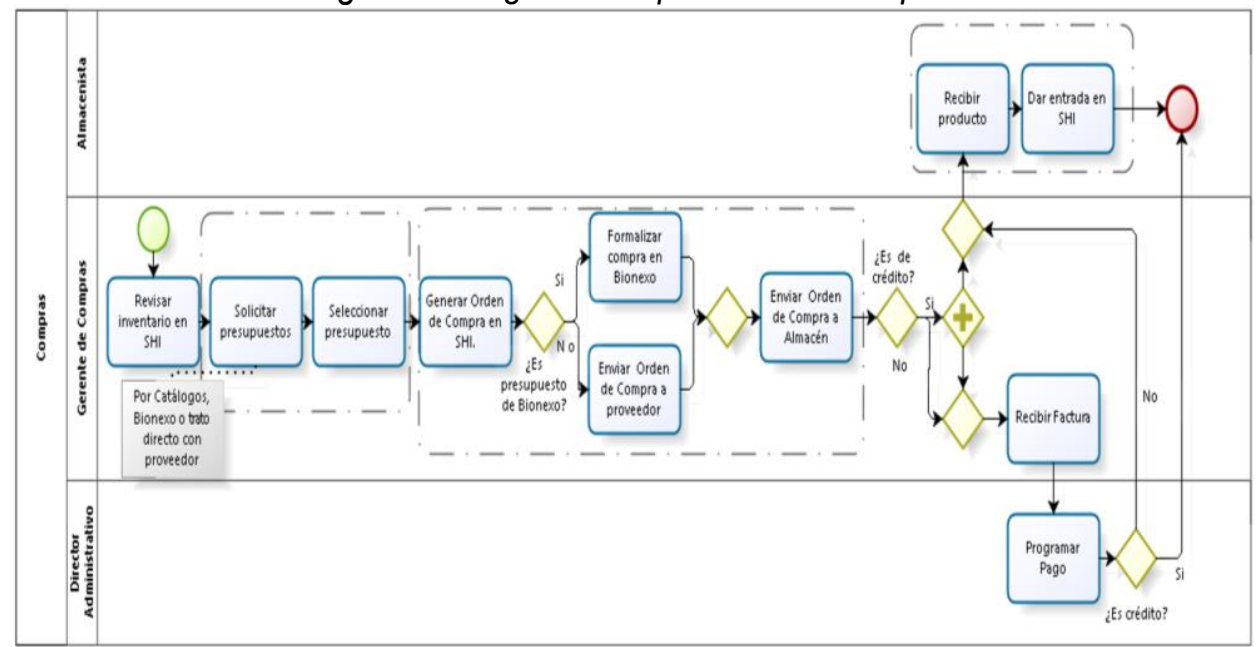

Fuente: Elaboración propia utilizando el software Bizagi modeler

Figura 2. Diagrama de proceso de compras especiales

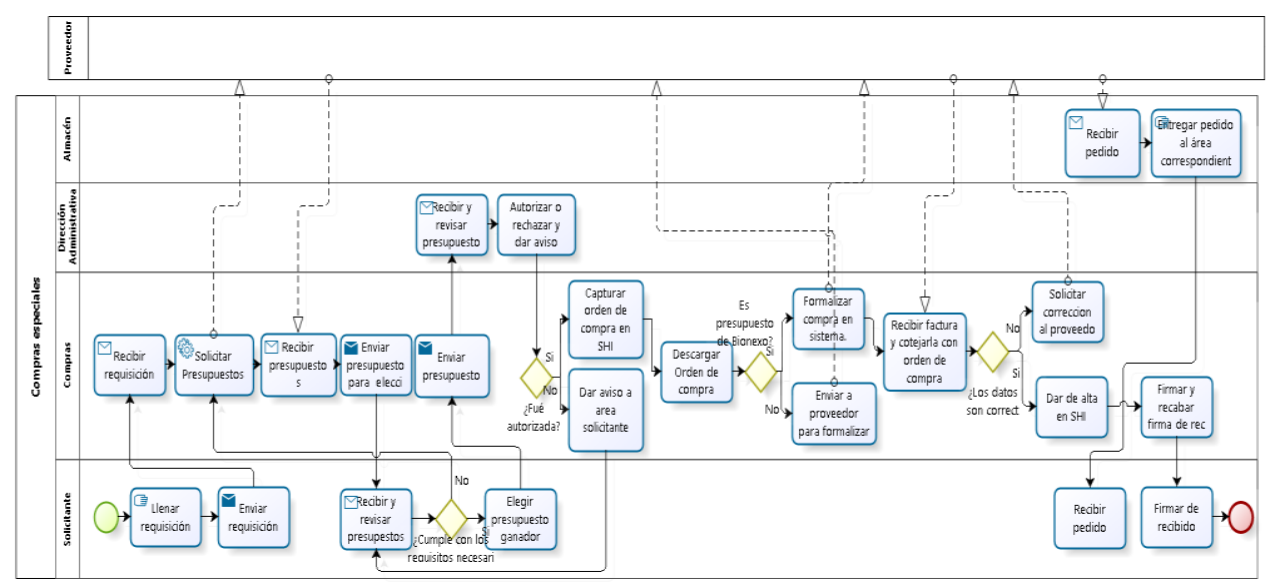

Fuente: Elaboración propia utilizando el software Bizagi modeler 
En la simulación y análisis del proceso real de compras especiales mostrado en la Figura 2, se obtuvo una duración de 9 días, 5 horas, 5 minutos, con un costo de $\$ 6,120.83$, este proceso presenta las mismas actividades que no generan valor del proceso de compras de cuadro básico, sumado a un tráfico de información mediante intermediarios, y esperas en autorizaciones de las cotizaciones y los presupuestos. De manera más específica se detectó lo siguiente:

- Las requisiciones son llenadas y entregadas de manera física y a mano, lo que ocasiona que el personal salga de su área de trabajo, y acumulación de papel para su traslado.

- Las cotizaciones se realizan de diferente manera, dependiendo del tipo de compra que se vaya a realizar, puede ser por sistema Bionexo 0 directamente con el proveedor.

- El llenado de orden de compra se realiza en físico y a mano, también es llenado digitalmente en el SHI, el cual tiene que ser descargado para luego enviarse al proveedor, en caso de no ser compra de Bionexo, en caso de ser compra de Bionexo es enviado directamente desde la plataforma al formalizar la compra aceptando el presupuesto del proveedor seleccionado como el mejor.

- $\quad$ En caso de compras especiales, una vez recibidas las cotizaciones son enviadas al área solicitante para su aprobación, una vez aprobada se le regresa al encargado de compras, y este la envía al director administrativo para su última aprobación, en caso de ser rechazado por alguno, se repite la operación con otra cotización. Esta parte del proceso genera muchos tiempos de espera, ya que el área solicitante o el director administrativo debido a su carga de trabajo pueden tardar de uno, dos o hasta tres días en contestar con aprobación, esto genera un retraso en lo consecutivo del proceso.

- Una vez realizada la compra, al llegar el producto la factura se coteja manualmente, revisando que todos los datos estén correctos y coincida con los datos especificados en la orden de compra, posteriormente se le da entrada al SHI.

- Para poder solicitar cotizaciones de medicamento del cuadro básico, primero se tiene que realizar una revisión de mínimos y máximos en $\mathrm{SHI}$, una vez seleccionados manualmente los productos que están en su mínimo, se

Fragoso, C., Borrego, G., Palacio R. \& Núñez, M. 
hace una lista para poder subirla al sistema Bionexo, todas estas actividades manuales requieren de tiempo y cuidado específico.

Con base en el análisis realizado se propone lo siguiente:

- Integrar módulo de requisiciones en Sistema Hospital Integral, para eliminar el formato en físico y el traslado del personal.

- Integrar solicitud de cotizaciones desde SHI para que todas las cotizaciones se realicen desde ese sistema. Además, agregar una restricción de tres cotizaciones para poder realizar orden de compra, así se asegura que realmente se estén solicitando cotizaciones a varios proveedores.

- Sistema de alertas en SHI, donde todos los involucrados estén informados de en qué estado se encuentra el proceso, quien está tardando en realizar lo que le corresponde y el mismo sistema esté mandando las alertas a las personas involucradas 0 responsables, de esos estados, y cuando llegue un nuevo formato 0 actividad.

- Automatizar la validación de factura electrónica con orden de compra como respaldo, dentro de SHI. La factura electrónica es un comprobante fiscal digital que se genera mediante internet (CFDI) se compone de dos documentos un XML y un PDF, es emitido a través del Servicio de Atención Tributaria en México para que se tenga registro de lo que una empresa o comerciante vende. La validación consistiría en anexar formato XML al recibirlo del cliente, y el sistema lo comparará con la orden de compra generada correspondiente a esa compra y con los datos fiscales de la clínica (Factura electrónica, s/f).

- Enlace desde SHI al sistema Bionexo, para eliminar los tiempos de revisión de inventarios y selección de producto por producto, con este enlace automáticamente se cargarían los productos que se encuentran en mínimos al sistema Bionexo para solicitar.

Se realizó el diagrama con la simulación de tiempos y costos aplicando los cambios propuestos mostrados a continuación en la Fig. 3 correspondiente al proceso de compras, y Fig.4 correspondiente al proceso de compras especiales. Al realizar la simulación se obtuvieron los resultados mostrados en la Tabla 2 donde se puede observar que los tiempos y costos disminuyeron en ambos procesos tal como los resultados obtenidos por 
Gómez (2013), por una vez que se realiza el proceso de compra de cuadro básico se reduce un $11 \%$ el costo de horas hombre y un $0.99 \%$ el tiempo total, cabe resaltar que es la reducción de una sola corrida del proceso el cual se realiza diariamente.

Figura 3. Diagrama proceso de compras propuesto

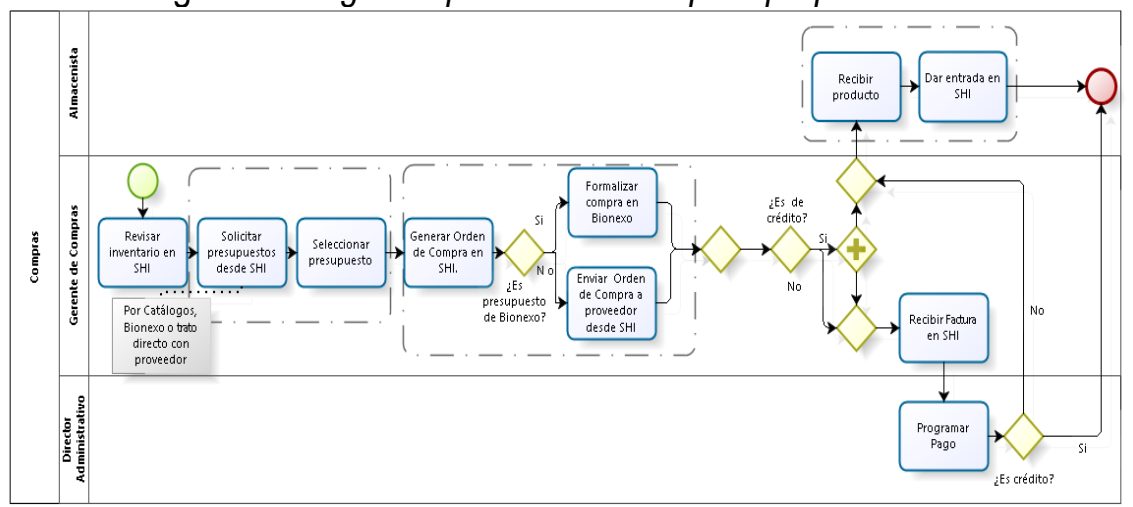

Fuente: Elaboración propia usando el software Bizagi modeler

Figura 4. Diagrama proceso de compras especiales propuesto

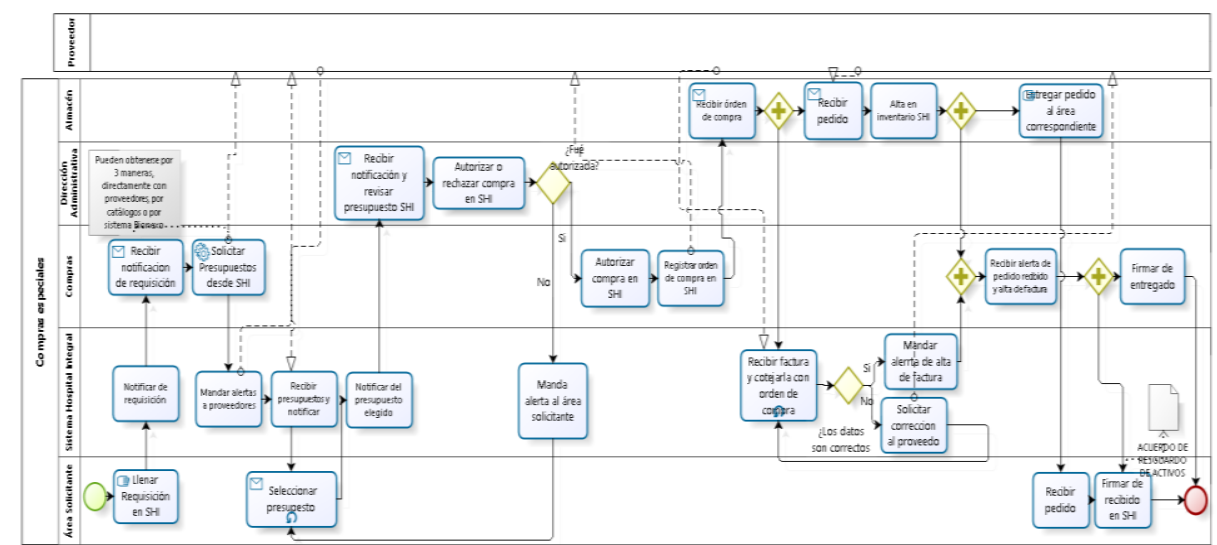

Fuente: Elaboración propia usando el software Bizagi modeler

Fragoso, C., Borrego, G., Palacio R. \& Núñez, M. 
Tabla 2. Tiempos y costos obtenidos en las fases de modelado y rediseño

\begin{tabular}{|c|c|c|c|c|c|}
\hline & & Proceso & Propuesta & Diferencia & $\begin{array}{c}\% \\
\text { producción }\end{array}$ \\
\hline \multirow{2}{*}{ Compras } & $\begin{array}{l}\text { Costo } \\
\text { Unitario }\end{array}$ & $\$ 2,833.33$ & $\$ 2,500.00$ & $\$ 333.33$ & $11.76 \%$ \\
\hline & $\begin{array}{l}\text { Tiempo total } \\
\text { Costo total }\end{array}$ & $\begin{array}{l}3 \mathrm{~d} 11 \mathrm{~h} 40 \mathrm{~m} \\
\$ 6,120.83\end{array}$ & $\begin{array}{c}3 \mathrm{~d} 10 \mathrm{~h} 50 \mathrm{~m} \\
\$ 3,634.03\end{array}$ & $\begin{array}{c}50 \mathrm{~m} \\
\$ 2,486.80\end{array}$ & $\begin{array}{c}.99 \% \\
40.62 \%\end{array}$ \\
\hline $\begin{array}{l}\text { Compras } \\
\text { especiales }\end{array}$ & Tiempo total & $9 d 5 h 5 m$ & $\begin{array}{c}7 \mathrm{~d} \text { 1h } 48 \mathrm{~m} \\
49 \mathrm{~s}\end{array}$ & $\begin{array}{c}2 \mathrm{~d} 3 \mathrm{~h} 16 \mathrm{~m} \\
49 \mathrm{~s}\end{array}$ & $23.29 \%$ \\
\hline
\end{tabular}

Fuente: Elaboración propia

Para el proceso de compras especiales se tiene una reducción de costos de horas hombre del $40.62 \%$ y un $23.29 \%$ del tiempo total.

\section{Guías de diseño}

Una vez realizada la simulación de las propuestas para el caso de estudio, esta sección se muestra una serie de recomendaciones generales de diseño obtenidas a partir del análisis de las necesidades del caso de estudio y del análisis de diferentes plataformas como Monday, BidDown y Wrike, aplicaciones que utilizan workflow como herramienta.

1) Línea del tiempo

Se propone que se muestre una línea del tiempo dónde cada persona que interviene en el proceso tenga acceso a ella, y pueda visualizar en qué punto se encuentra el proceso, y el estado de cada actividad dentro de él, con ello se tendría información clara para cualquier interesado en el proceso y sobre todo detectado siempre quien es la persona responsable de que el proceso esté detenido en caso de que esto sucediera. Por ejemplo, en el proceso estudiado de compras especiales hay dos actividades donde se tiene que esperar autorización de la actividad, en caso de que al responsable se le olvide autorizar, pude retrasarse días la llegada del producto a comprar, sin la información de la línea del tiempo, nadie tiene conocimiento del retraso, ni el motivo, ni el responsable de ello.

\section{2) Cronograma}

Agregar fechas de inicio y plazos específicos para las actividades que lo requieran, así como las vistas del cronograma del proceso. Con este punto es donde se especifica a cada responsable de la actividad, cuanto tiempo tiene para realizarla, y todos los integrantes e interesados del proceso tienen visibilidad de ello. 
3) Prioridad

Facilidad de agregar grados de prioridad tanto al proceso como a cada actividad: Alta, media, o baja. Con ello todos los responsables del proceso tienen conocimiento de la prioridad que debe tomar su actividad, debido a que no todas las compras son del mismo producto ni tienen la misma prioridad, incluso un mismo producto puede cambiar de prioridad dependiendo la situación.

4) Asignar tareas

Asignar un responsable a cada actividad, dónde automáticamente se le envía una notificación con la información relevante (prioridad y plazos) al momento de la asignación. En el proceso estudiado las personas tienen sus actividades asignadas informalmente, ellos saben lo que tienen que hacer, pero no hay ningún documento 0 sistema que se los estipule, al momento de asignar las tareas en el sistema queda registrado el responsable de cada actividad, sobre todo porque a excepción del encargado de compras para los demás involucrados este proceso no se encuentra entre sus actividades primordiales, por lo que pueden ir dejando pasar su responsabilidad dentro del proceso de compras y retrasarlo.

5) Comunicaciones

Interfaz y automatización para el envío de documentos, alertas, menciones y mensajes con opciones de respuesta. Esto ayudará a disminuir tiempos en la comunicación, eliminar traslados, y documentación en físico. En el proceso estudiado se entregan formatos en físico, hay actividades de espera por autorización en la que si no se obtiene respuesta es necesario enviar un correo o realizar una llamada para apurar la respuesta por parte de la persona encargada de autorizar para poder continuar con el proceso, es lo que se busca eliminar con este punto.

6) Integración

Habilitar integración con otras aplicaciones, plataformas o herramientas de gestión y comunicación (correo electrónico, plataformas de proveedores, etc.), para que dentro del mismo sistema se puedan recibir y enviar correos, documentos, y mensajes sin tener que salir de él. En el proceso se observó que hay muchas actividades donde el responsable de compras tiene que descargar documentos como las cotizaciones, órdenes de compra o facturas, para después enviarlas por correo a quien corresponda, con este punto

Fragoso, C., Borrego, G., Palacio R. \& Núñez, M. 
podría enviarlo directamente desde el sistema a la plataforma que más convenga para el proceso, ya sea correo electrónico o plataforma del proveedor, por mencionar algunos. Logrando así centralizar la información del departamento de compras en el sistema, que es lo que ofrecen todas las plataformas analizadas.

7) Catálogo de proveedores

Crear una agenda homologada de proveedores, dónde se puedan agregar y evaluar aspectos de interés para el proceso de compra (producto, satisfacción, condiciones de pago, porcentajes de descuentos, etc). Actualmente en el proceso cuentan con una agenda de proveedores, pero lo que desconocen son los porcentajes de descuento con los que cuentan con cada uno, o si ya consumen el estimado necesario para acceder a un descuento mayor, ya que los proveedores manejan su descuento por cantidad de consumo.

8) Cotizaciones y órdenes de compra

Poder solicitar cotizaciones y enviar órdenes de compra a los proveedores desde el sistema, así como crear un historial y seguimiento de ellas. Para el proceso de compras estudiado, no se cuenta con un sub proceso estandarizado, ya que las cotizaciones que se realizan directamente con el proveedor, por la plataforma Bionexo, o por los catálogos, no se cuenta con un registro de ellos, y en el caso de las compras especiales, solo se guarda cuando el producto comprado tiene un costo muy elevado. En el caso de las órdenes de compras como antes se mencionó, se realizan en formato en físico y después en el sistema. Al implementar la cotización y orden de compra desde el sistema se contará con el historial de cada una de ellas, y en caso de ser necesario dar seguimiento.

9) Agenda electrónica

Facilidad de cargar agenda de contactos, y de invitar a través del sistema aquellos contactos que desee.

10) Reportes

Posibilidad de generar reportes de todo aquello que ocurre en los procesos, poder visualizar el progreso de cada tarea, comparar resultados con resultados anteriores, presupuestos, descuentos obtenidos, cronogramas, líneas del tiempo, etc. Este apartado es uno de los principales elementos de la plataforma Monday, dónde se hace recuento de todo lo realizado, y se encuentra a disposición de los interesados, porcentajes, 
tiempos totales, comparación entre procesos realizados, tiempos de espera, toda la información importante para la toma de decisiones y posibles mejoras del proceso.

Con los puntos mencionados de la guía de diseño se cumplen a los beneficios que muestra Gómez (2013) en su estudio, donde al implementar el sistema workflow se mejora la gestión de compras, logrando automatizar los procesos de trabajo, y así generar control en todas las etapas del proceso dónde los documentos y la información se mueven entre los involucrados, reduciendo costos y aumentando el rendimiento de los trabajadores ya que tareas como la búsqueda, reportes para análisis, envío de documentos, pasa ahora a manos del sistema workflow. Además cumpliendo con una buena gestión de compras la empresa puede responder a demandas cambiantes y resaltar en más beneficios como lo menciona Andino (2006).

\section{Conclusiones}

En este artículo se presentó un estudio siguiendo la metodología PADM con los que se obtuvieron resultados de cómo se encontraba el proceso de compras, que llevaron a un análisis de herramientas en el mercado que establecen un flujo de trabajo donde se automatizan los procesos, se asignan tareas y prioridad de ellas, se integran aplicaciones, contando con alertas y con acceso la información de manera sencilla y en tiempo real, eliminando documentación en físico, así como poder visualizar el progreso de cada proceso; los cuales en estos momentos no se toman en cuenta y son vitales para optimizar los procesos a los que se apliquen.

Con las guía de diseño propuestas, la clínica podría implementar un sistema de flujo de trabajo en su proceso de compras, logrando disminuir tiempos y costos tal como se menciona en el trabajo de Gómez (2013). Las guías de diseño se obtuvieron a partir de los resultados y analizando otras herramientas en el mercado que apoyan la gestión de compras, de las que se obtuvieron los requerimientos del sistema de acuerdo al proceso de compras de una clínica privada.

Con la metodología PADM se pudo documentar y mapear el proceso, para analizar y detectar las mejoras que pudieran implementarse y crear un flujo de trabajo, automatizando los procesos y generando control en las diferentes etapas donde los documentos y la información se mueven entre

Fragoso, C., Borrego, G., Palacio R. \& Núñez, M. 
los interesados (Gómez, 2013), integrándolas en un único entorno, lo cual ayuda a las organizaciones a crear estructuras de adaptación tecnológica para prosperar ante cambios o evoluciones en el mercado (Haposan et al., 2020).

El objetivo de las guías presentadas fue identificar y establecer los principales requerimientos para un flujo de trabajo en el proceso de compras de un hospital privado y sus beneficios. A parte de beneficiar directamente al proceso de compras y a las personas involucradas, la clínica puede obtener más beneficios en otras áreas, como seguridad para los doctores y pacientes de que lo que receten siempre se encontrará disponible en la farmacia de la clínica, un orden y disponibilidad de documentos y alertas para que se realicen los pagos a los proveedores en tiempo y forma, un mayor flujo de efectivo ya que con el seguimiento y comparación entre proveedores se obtienen mayores descuentos, por lo tanto menor gasto y mayor ganancia en el medicamento.

Las guías de diseño presentadas pueden ser aplicada a otros hospitales privados sin necesidad de repetir la metodología PADM, incluso como trabajo futuro se pretende presentar para otros procesos, así como la implementación de la guía, primero elaborando las interfaces para que los usuarios la evalúen, realizando las correcciones en caso de necesitarlas y así poder con la implementación, seguimiento y análisis del sistema, lo que tendría un impacto exponencial en el hospital que lo implemente.

Las guías de diseño son una oportunidad para los hospitales privados de contar con un recurso en el cual basarse para comenzar con la automatización de sus procesos en la que encontrarán los elementos principales para lograr un flujo de trabajo y el beneficio que cada uno representa, otra oportunidad de estudio sería para el caso de hospitales públicos ya que los hospitales públicos realizan sus compras cada año con un proceso denominado compras consolidadas, las cuales se realizan por licitaciones y adjudicaciones, en el cual interviene el gobierno de México, la política de combate a la corrupción, la Oficialía Mayor de la Secretaría de Hacienda y Crédito Público, en coordinación con el Sector Salud (Presidencia de la República, 2019).

\section{Referencias}

Andino, R. (2006). Gestión de inventarios y compras. En eoi Escuela de Negocios (p. 73). 
http://api.eoi.es/api_v1_dev.php/fedora/asset/eoi:45152/componente45150.pdf

Beltrán, B. A. G., \& García, A. G. (2018). Guías De Diseño Web Para Facilitar El Acceso a La Información Desde Teléfonos Inteligentes. Pistas Educativas, 39(128), 586-606. http://www.itcelaya.edu.mx/ojs/index.php/pistas/article/view/1183

Camacho, H. C., Lorena, K., Espinosa, G., \& Arboleda, U. S. (2012). Importancia de la cadena de suministros en las organizaciones. LACCEI, 10.

Conceição, P., \& Gonzalez, F. (2010). Maximizing the benefits of ERP systems. Revista de Gestão da Tecnologia e Sistemas de Informação, 7(1), 5-32. https://doi.org/10.4301/S1807-17752010000100001

Dhir, S. (2018). Flexible Strategies in VUCA markets (S. Dhir (ed.)). Springer. https://doi.org/https://doi.org/10.1007/978-981-10-8926-8

Factura electrónica. (s/f).

Freund, J., Rücker, B., \& Hitpass, B. (2017). BPMN Manual de Referencia y Guía Práctica (5a ed.).

Gómez, V. (2013). La implantación de un sistema workflow para la mejora de gestión de compras del grupo ANECOOP. http://hdl.handle.net/10251/35076

Haposan, C., Husseini, F., Barkah, A., Prihatma, O., \& Putra, B. (2020). An analysis on the importance of motivation to transfer learning in VUCA environments. Management Science Letters, 10, 271-278. https://doi.org/10.5267/j.msl.2019.9.005

Hernández, R., Fernández, C., \& Baptista, M. del P. (2014). Metodología de la investigación

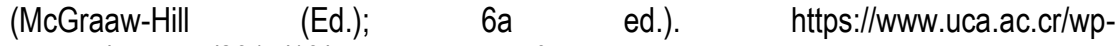
content/uploads/2017/10/Investigacion.pdf

Inglés-Romero, J. F., \& Vicente-Chicote, C. (2012). Guías de diseño para sistemas adaptativos basados en componentes. $V$ Jornadas de introducción a la investigación de la UPCT, 96-98. http://repositorio.upct.es/handle/10317/3268

Instituto Mexicano del Seguro Social. (s/f). Medicamentos. http://www.imss.gob.mx/profesionales-salud/cuadros-basicos/medicamentos

Jiang, J. S., Yang, B., \& Miao, Z. M. (2015). A workflow task assignment method based on the properties of task and user. Computer Simulation, 32, 222-225. https://www.jstor.org/stable/26543031?read-now=1\&seq=5\#page_scan_tab_contents

Jiménez, A. P., Salamanca, P. A., \& Garavito, L. A. (2007). Sistema de información orientado a procesos de negocio y flujos de trabajo en la Universidad Nacional de Colombia . Perspectivas y caso de estudio A business management- and workflow-orientated information system in the Universidad Nacional de Colombia: Ingeniería e investigación, 27(3), 193-202.

Martinez, A., \& Cegarra, J. G. (2014). Gestión por procesos de negocio: organización horizontal.

Montoya, A. (2002). Conceptos modernos de administración de compras (Norma (Ed.); 19a ed.). http://xurl.es/zq5bq

Muñiz, L., \& Prat, R. (2003). La implantación de sistemas tipo ERP: Su efecto sobre la organización y los recursos humanos. Partida doble, 150, 32-45.

Orellana, S. D. G. (2018). Evaluación de las compras corporativas de medicamentos en el sector salud , Lima 2014 - 2016 [Universidad César Vallejo].

Fragoso, C., Borrego, G., Palacio R. \& Núñez, M. 
http://repositorio.ucv.edu.pe/bitstream/handle/UCV/12986/Orellana_YSDG.pdf?sequen $\mathrm{ce}=1$ \&isAllowed=y

Presidencia de la República, P. (2019). Compra consolidada de medicamentos y material de curación 2020.2 [Comunicado prensa]. https://www.gob.mx/presidencia/prensa/compra-consolidada-de-medicamentos-ymaterial-de-curacion-2020

Rodriguez, J., \& González, J. (2002). Integración de las tecnologías de flujo de trabajo y gestión documental para la optimización de los procesos de negocio. Ciencias de la Información, 33(3), 17-28. https://docplayer.es/7244726-Integracion-de-lastecnologias-de-flujo-de-trabajo-y-gestion-documental-para-la-optimizacion-de-losprocesos-de-negocio.html

Salas, G., \& Campoverde, M. (2019). Proceso de Gestión de Asistencia Técnica. Caso de estudio: Hospital General Isidro Ayora de Loja, Ecuador. Dominio de las ciencias, 5, 159-183. https://doi.org/10.23857/dc.v5i3.930 